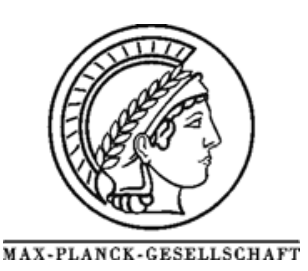

\title{
Intensities of combination IR bands as an indication of a concerted mechanism of proton transfer from acidic hydroxyl groups in zeolites to the ethylene hydrogen-bonded by proton
}

\author{
V.B. Kazansky*,1, I.R. Subbotina ${ }^{1}$, F.C. Jentoft ${ }^{2}$ \\ ${ }^{1}$ Zelinsky Institute of Organic chemistry, Leninsky prospect 47, Moscow 119991, Russia. \\ ${ }^{2}$ Department of Inorganic Chemistry, Fritz-Haber-Institute of the MPG, Faradayweg 4-6, 14195 Berlin, Germany \\ *Corresponding author: e-mail vbk@ioc.ac.ru,
}

Received: 4 October 2005; revised 26 December 2005; accepted 22 February 2006

\begin{abstract}
Adsorption of ethylene by sodium forms of mordenite and $\mathrm{Y}$ zeolite is reversible and results in no chemical activation of olefin. Therefore, diffuse reflectance infrared Fourier transform spectra of ethylene adsorbed by these zeolites do not differ much from those of liquefied or frozen $\mathrm{C}_{2} \mathrm{H}_{4}$. In contrast, adsorption of ethylene by HY and HMOR results in the strong hydrogen bonding with acidic hydroxyl groups and in subsequent oligomerization of ethylene already at room temperature. The hydrogen bonding strongly perturbs $\mathrm{OH}$ frequencies but exerts only a weak influence on the frequencies and intensities of the fundamental C-H stretching vibrations of ethylene. In contrast, intensities of the infrared (IR) bands from combinations of the double bond stretching vibrations with the bending vibrations of $\mathrm{CH}_{2}$ groups increase significantly. According to previously published quantum chemical calculations, these combinations contribute most significantly to the reaction coordinates of protons added to adsorbed ethylene. Therefore, the results obtained allow formulation of a new spectral criterion of the reactivity index for concerted proton transfer to adsorbed ethylene; the IR bands corresponding to combination frequencies of most strongly polarized chemical bonds involved in this elementary step have unusually high intensities. It is also suggested that this criterion is of more general significance and also can be applied to other elementary steps of acid or acid-base catalytic reactions when chemical activation of adsorbed molecules results from simultaneous polarization of several chemical bonds.
\end{abstract}

Keywords: reactivity index, reaction coordinate, combinations of stretching and bending vibrations, acid catalysis, zeolites, IR spectra.

\section{Introduction}

Infrared (IR) spectroscopy is most frequently used for the studing the mechanism of acid heterogeneous catalysis. It allows both observation of perturbation of acidic hydroxyl groups via their hydrogen bonding with adsorbed molecules and detection of the stable protonated active intermediates. The low-frequency shifts of IR stretching bands of hydroxyl groups or adsorbed molecules are also frequently used as a measure of their perturbation and chemical activation. However, for adsorbed hydrocarbons, in most cases such shifts do not exceed only few wave numbers [1]. This finding can be explained by the covalent nature of the ground states of $\mathrm{C}-\mathrm{H}$ chemical bonds. Thus, the frequencies of their fundamental stretching vibrations are rather insensitive to polarization of chemical bonds, which is the main source of chemical activation of adsorbed hydrocarbons in heterogeneous acid catalysis.

Bearing this in mind, in previous work $[2,3]$ we proposed using intensities of C-H IR stretching bands as measures of the polarization of adsorbed light paraffins by the low-coordinated cations in zeolites. The obvious advantage of this new spectral approach lies in the closer connection of the intensities of IR bands with polarization of C-H chemical bonds on stretching via vibrational excitation. Indeed, it is well known from the fundamentals of IR spectroscopy that intensities of the stretching bands of different molecules are proportional to the squares of the first derivatives of additional dipole moments, $\mathrm{d} \mu$, created by the stretching of chemical bonds over normal coordinate, $q$, of the corresponding vibrations: 


$$
\mathrm{I}_{\text {fund }} \sim[\mathrm{d} \mu / \mathrm{dq}]^{2}
$$

In other words, the intensities of IR stretching bands allow much better tracing polarization of adsorbed molecules by the active sites of heterogeneous catalysts than do the low frequency shifts of these vibrations. For the acid and acid-base catalysis, when chemical activation of adsorbed molecules mainly arises from their polarization, additional application of this approach seems promising.

In the present study we attempted to apply this new spectral criterion to another kind of acid-catalyzed reaction: to ethylene oligomerisation over hydrogen forms of mordenite and Y zeolite. To do this, we compared IR spectra of ethylene adsorbed by the hydrogen and sodium forms of these zeolites.

The reason that we chose these zeolites and ethylene oligomerisation as a test reaction is that ethylene is one of the simplest molecules, and thus its IR spectrum can be analyzed in details rather easily. In addition, although ethylene adsorption on both sodium and hydrogen forms of the zeolites starts with formation of similar $\pi$-complexes, for the sodium forms it does not result in subsequent chemical activation and oligomerisation. In contrast, ethylene $\pi$ bonded to acidic protons is iniitially transformed into surface ethoxy groups and then involved in subsequent oligomerization with formation of the growing polyethylene chains anchored to the surface of the zeolites [4,5]. Therefore, such differing ethylene activation by the hydrogen and sodium forms could be also expected to affect the IR spectra of the $\pi$-bonded species.

Another argument in favor of acid-catalyzed ethylene oligomerization as a test reaction was provided by our earlier quantum chemical calculations [6-8]. These calculations described the mechanism of proton transfer to the $\pi$ bonded olefins reasonably well; therefore, we expected that compariing of the results of quantum chemical calculations with those of the present spectral study could provide insight into the role of the polarization of $\pi$-bonded olefins in the reaction mechanism of proton transfer.

Our measurements of IR spectra of adsorbed ethylene were done by the highly sensitive diffuse reflection ifrared Fourier transforming spectroscopy (DRIFTS) method. The spectral range studied included the frequencies of C-H stretching vibrations and combinations of double bond stretching with bending vibrations of $\mathrm{CH}_{2}$ groups. Hydrogen forms of Y zeolite and mordenite with Broensted acid sites of varying strengths were selected as the solid catalysts. The dimensions of the micro pores in these zeolites are also varied somewhat. However, in the present study we did not explore the role of the shape selectivity in the process of ethylene oligomerization, but rather concentrated on the primary key reaction of the addition protons to ethylene.

\section{Experimental}

Y zeolite from "Akzo Chemical" used in the present study had a $\mathrm{Si} / \mathrm{Al}$ ratio equal to 2.5 , while for mordenite this ratio was equal to 5 . Five-fold ion exchange of sodium forms was carried out with an ammonium nitrate aqueous solution. After ion exchange the catalysts were washed with distilled water and dried for five hours at $300 \mathrm{~K}$. The hydrogen forms of both zeolites were prepared by decomposition of the corresponding ammonium forms in an oxygen flow at $700 \mathrm{~K}$. The extent of sodium substitution as detected by AAS analysis was as high as $90-95 \%$.

For preparation of the deuterated mordenite, the hydrogen form was kept in vapors of the heavy water for 30 min at $600 \mathrm{~K}$. As follows from DRIFT spectrum of the deuterated sample, the extent of hydrogen substitution by deuterium was close to $100 \%$.

For the spectral measurements the granulated samples of both zeolites with particle size of $0.5-1 \mathrm{~mm}$ were placed in a quartz vacuum cell equipped with the side arm with a $\mathrm{CaF}_{2}$ window transparent for IR irradiation. The vacuum pretreatment of the zeolites was carried out in the quartz part of the cell. At first the samples were heated in vacuum at $100 \mathrm{C}$ for $2 \mathrm{~h}$. Then the temperature was gradually increased from 373 to $723 \mathrm{~K}$, and the samples were maintained at this temperature under vacuum for 3 more hours.

DRIFT spectra were recorded at room temperature in the spectral range of $2000-4000 \mathrm{~cm}^{-1}$ with a Nicolet Impact 410 spectrophotometer equipped with a home-made DRIFT chamber. For this purpose the granulated powders of the zeolites were transferred to the side arm of the optical vacuum cell with a $\mathrm{CaF}_{2}$ window. The technique of these measurements is described in more details in Ref [9]. The obtained spectra were converted into Kubelka-Munk units using a standard program under assumption, that the reflective ability of the zeolites at $5000 \mathrm{~cm}^{-1}$ was equal to 0.9 . The backgrounds created by the zeolites were then subtracted from the total spectra.

Prior to adsorption, ethylene was purified by freezing in a trap cooled with liquid nitrogen followed by selection of the middle fraction that was evaporated upon gradual increased temperature. To avoid fast oligomerisation over hydrogen form of mordenite (HMOR), ethylene was initially adsorbed on this zeolite in the amount of 3-7 mmol/g at $77 \mathrm{~K}$. To make distribution of ethylene inside the micropores of the zeolites more homogeneous, the closed vacuum cell with samples was slowly warmed to $220 \mathrm{~K}$, then again cooled down to $77 \mathrm{~K}$ and brought in a Dewar flask under liquid nitrogen to the IR spectrophotometer. After warming the cell to the room temperature, the zeolite granules were rapidly transferred into its side arm with $\mathrm{CaF}_{2}$ window, and DRIFT measurements were started with the intervals of several minutes between the subsequent recordings. Ethylene adsorption on hydrogen and sodium forms of $\mathrm{Y}$ zeolite and on sodium form of mordenite was carried out in the same optical vacuum cell directly at room temperature at the pressure of 8-10 torr. 
Table 1: Wavenumbers of IR C-H stretching and combination bands for gaseous, liquified, solid ethylene and for ethylene adsorbed by sodium and hydrogen forms of $\mathrm{Y}$ zeolite and mordenite.

\begin{tabular}{|c|c|c|c|c|c|c|c|}
\hline $\begin{array}{l}\text { Assignement } \\
\text { of IR absorp- } \\
\text { tion bands } \\
\text { according to } \\
{[10]}\end{array}$ & $\begin{array}{c}\text { Gaseous } \\
\mathrm{C}_{2} \mathrm{H}_{4}\end{array}$ & $\begin{array}{l}\text { Liquified } \\
\mathrm{C}_{2} \mathrm{H}_{4} \text { at } \\
-168^{0} \mathrm{C}^{*}\end{array}$ & $\begin{array}{c}\text { Solid } \\
\mathrm{C}_{2} \mathrm{H}_{4} \text { at } \\
-180^{0} \mathrm{C}^{*}\end{array}$ & $\begin{array}{c}\mathrm{C}_{2} \mathrm{H}_{4} \text { ad- } \\
\text { sorbed by } \\
\mathrm{NaY}^{* *}\end{array}$ & $\begin{array}{c}\mathrm{C}_{2} \mathrm{H}_{4} \text { ad- } \\
\text { sorbed by } \\
\text { NaMOR** }\end{array}$ & $\begin{array}{c}\mathrm{C}_{2} \mathrm{H}_{4} \text { ad- } \\
\text { sorbed by } \\
\mathrm{HY}^{* *}\end{array}$ & $\begin{array}{l}\mathrm{C}_{2} \mathrm{H}_{4} \text { ad- } \\
\text { sorbed by } \\
\mathrm{HMOR}^{* *}\end{array}$ \\
\hline$v_{1}$ & 3026 (R) & 3016 & - & 2995 & 2997 & 3003 & 3003 \\
\hline$v_{5}$ & 3103 (R) & 3075 (R) & 3075 & 3019 & - & - & 3065 \\
\hline$v_{9}$ & 3105 & 3085 & & 3085 & 3082 & 3092 & 3094 \\
\hline$v_{11}$ & 2989 & 2983 & 2973 & 2972 & 2969 & 2975 & 2975 \\
\hline$v_{2}$ & $1623(\mathrm{R})$ & 1620 & 1616 & & & & \\
\hline$v_{3}$ & $1342(\mathrm{R})$ & 1339 & 1336 & & & & \\
\hline$v_{6}$ & - & 1239 & - & & & & \\
\hline$v_{4}$ & 1027 & - & 1010 & & & & \\
\hline$v_{7}$ & 949 & 961 & 970 & & & & \\
\hline$v_{10}$ & 810 & 828 & 827 & & & & \\
\hline$v_{2}+v_{10}$ & $\begin{array}{l}- \\
-\end{array}$ & 2434 & 2434 & 2427 & 2421 & $\begin{array}{c}\text { broad bands } \\
2351\end{array}$ & $\begin{array}{c}\text { broad bands } \\
2353\end{array}$ \\
\hline$v_{3}+v_{4}$ & - & 2340 & - & 2353 & 2362 & & \\
\hline$v_{3}+v_{7}$ & $\begin{array}{l}- \\
-\end{array}$ & - & 2300 & 2277 & 2273 & 2272 & 2267 \\
\hline $\begin{array}{l}v_{4}+v_{6} \\
v_{3}+v_{10}\end{array}$ & - & $\begin{array}{l}2253 \\
2167\end{array}$ & $\begin{array}{l}2255 \\
2166\end{array}$ & 2163 & 2166 & 2167 & 2167 \\
\hline
\end{tabular}

* C.Brecher, R.S.Halford, J. Chem.Phys. 35(1961)1109.

** Present work $\left(v_{2}, v_{3}, v_{6}, v_{4}, v_{7}, v_{10}\right.$ were not recorded $)$

\section{Experimental Results}

\section{a) Adsorption of ethylene by sodium forms of NaY and NaMOR}

Adsorption of ethylene at room temperature by NaMOR and $\mathrm{NaY}$ zeolites is reversible and does not result in any chemical reaction. The corresponding DRIFT spectra are shown in Figs. 1 (a) and 2 (a). In addition, the comparison of the wave numbers of the bands of adsorbed species with those for gaseous or frozen ethylene is given in Table 1.

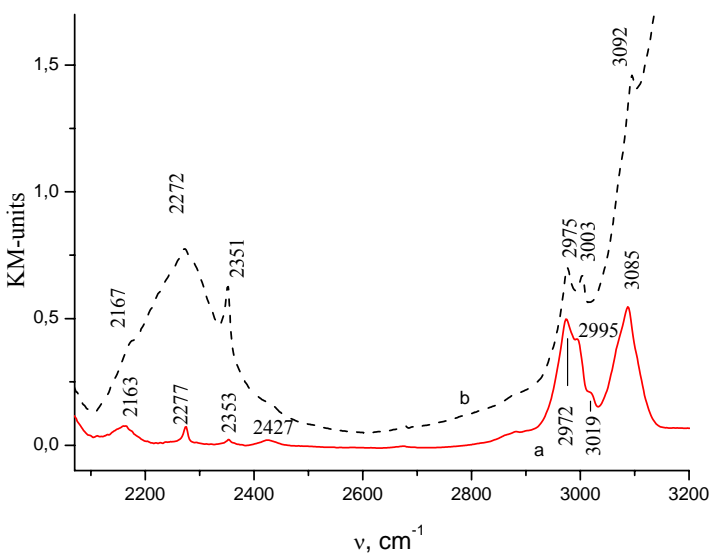

Figure 1: DRIFT spectra of ethylene adsorbed by $\mathrm{NaY}$ (a) and $\mathrm{HY}(\mathrm{b})$ zeolites at room temperature at the pressure of 8 torr and 10 torr respectively.

For ethylene adsorbed by both sodium forms, the frequencies of $v_{9}$ and $v_{11}$ asymmetric $\mathrm{C}-\mathrm{H}$ stretching bands are very close to each other and to those in the spectra of gaseous, liquid or frozen ethylene. In addition, we also observed the symmetry forbidden $v_{1}$ and $v_{5} \mathrm{C}$-H stretching bands that are absent in the spectrum of gaseous ethylene. This well-known effect is connected with the lowering of the symmetry of adsorbed molecules due to their interaction with the adsorption sites of zeolites or other adsorbents [1]

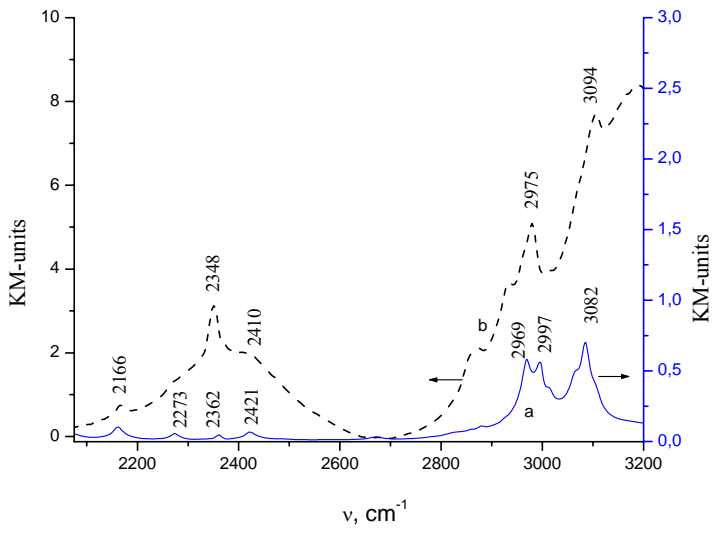

Figure 2: (a) DRIFT spectra of ethylene adsorbed by NaMOR at room temperature at the pressure of 10 torr. (b) 
DRIFT spectrum of HMOR, recorded at room temperature in $0.5 \mathrm{~min}$. after adsorption of ethylene in amount of 7 $\mathrm{mmol} / \mathrm{g}$

As follows from Table 1, for both sodium forms the positions of the combination IR bands of different bending vibrations with the stretching vibrations of the double bond in the spectral range of $2100-2500 \mathrm{~cm}^{-1}$ are also very close to each other. They are also very close to those in the spectra of liquid or frozen ethylene. As one may expect, the intensities of these bands are about one order of magnitude lower than those of the $\mathrm{C}-\mathrm{H}$ stretching bands. Thus, the interaction of ethylene with sodium forms of both zeolites neither results in chemical activation, nor in large lowfrequency shifts of the IR absorption bands.

According to Ref. [10], the above combinations most likely arise from interaction of the $v_{3}\left(1342 \mathrm{~cm}^{-1}\right)$ and $v_{2}$ $\left(1623 \mathrm{~cm}^{-1}\right)$ stretching vibrations of the double bond with the $v_{4}\left(1027 \mathrm{~cm}^{-1}\right), v_{7}\left(949 \mathrm{~cm}^{-1}\right)$ or $v_{10}\left(810 \mathrm{~cm}^{-1}\right)$ twisting and $\mathrm{CH}_{2}$ bending vibrations.

\section{b) Adsorption and oligomerization of ethylene on hydrogen form of $Y$ zeolite.}

As has been earlier reported [11], ethylene adsorption by the hydrogen forms of zeolites results in the strong broadening and low-frequency shifts of $\mathrm{O}-\mathrm{H}$ stretching bands of acidic hydroxyl groups. In our case, for ethylene adsorption on $\mathrm{HY}$ at the equilibrium pressure of 10 torr the maximum of this band shifts from 3660 to $3285 \mathrm{~cm}^{-1}$. In addition, the weak $\mathrm{C}-\mathrm{H}$ stretching bands of adsorbed ethylene at 3092,3003 and $2875 \mathrm{~cm}^{-1}$ are superimposed on the background of this broad band (Fig. 1 b). Similar to adsorption by $\mathrm{NaY}$, the positions of these bands differ only slightly from those for frozen or liquefied ethylene. Thus, adsorption of ethylene on HY does not perturb considerably the bands from the $\mathrm{C}-\mathrm{H}$ stretching vibrations.

In contrast, comparison of the spectra (a) and (b) in Fig. 1 reveals that in the spectral range of combinations of different bending vibrations with the stretching vibrations of the $\mathrm{C}=\mathrm{C}$ double bond, there is a huge difference between DRIFT spectra of ethylene adsorbed by $\mathrm{NaY}$ and $\mathrm{HY}$ zeolites. Indeed, for ethylene adsorption by $\mathrm{NaY}$, this part of IR spectrum displays four well-resolved weak narrow bands (spectrum (a) in Fig. 1 ). In contrast, for ethylene adsorbed by HY only a narrow band with a maximum slightly shifted to $2351 \mathrm{~cm}^{-1}$ is detected. The intensity of this band strongly increases and becomes comparable with those of the C-H stretching bands. The other combination bands are transformed into a very broad unresolved line with a maximum at about $2400 \mathrm{~cm}^{-1}$. The intensity of this line is by more than two orders of magnitude higher than that of the corresponding narrow bands for ethylene adsorbed by the sodium form (spectrum (b) in Fig. 1 ).

Indeed, for ethylene adsorbed by $\mathrm{NaY}$ zeolite, the amplitudes of the combination bands are about one order of magnitude lower than for the bands from $\mathrm{C}-\mathrm{H}$ stretching vibrations, while for the hydrogen bonded ethylene, the amplitude of the broad band is comparable with those of the $\mathrm{C}-\mathrm{H}$ stretching bands. In addition, this new combination band is more than by one order of magnitude broader. Therefore, the hydrogen bonding of ethylene with acidic hydroxyl groups results in very much higher intensity of the bands from the combinations of the double bond stretching vibration with the bending vibrations of $\mathrm{CH}_{2}$ groups.

There is no doubt that this effect is related to chemical activation of adsorbed molecules, since keeping of HY zeolite in the ethylene atmosphere at room temperature results in a very slow oligomerization. In Fig. 3 this reaction is indicated by appearance of the new $\mathrm{C}-\mathrm{H}$ stretching bands. Two of them at 2865 and $2930 \mathrm{~cm}^{-1}$ correspond to symmetric and asymmetric $\mathrm{CH}_{2}$ stretching vibrations of the growing oligomer chains, while the band with the maximum at $2960 \mathrm{~cm}^{-1}$ belongs to the asymmetric vibration of $\mathrm{CH}_{3}$ groups. These bands could not be removed by evacuation at room temperature.

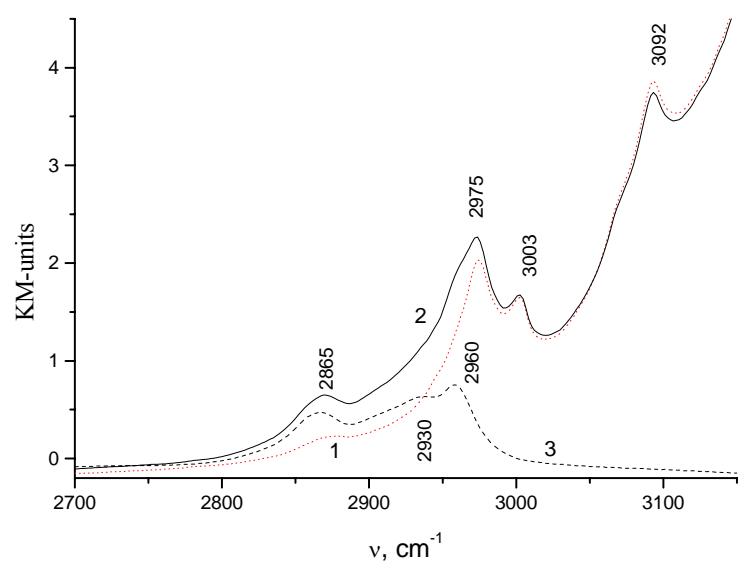

Figure 3: DRIFT spectrum of ethylene adsorbed by HY at room temperature at the pressure of 200 torr after 1 (1) and 50 (2) hours from the beginning of the reaction. Spectrum (3) was recorded after evacuation of the sample after reaction at room temperature.

\section{c) Adsorption and oligomerization of ethylene on the hydrogen form of mordenite.}

The results obtained in the present work for ethylene adsorption by hydrogen form of mordenite are very similar to those for adsorption by HY. This is illustrated by the DRIFT spectra, shown in Fig. 4. They indicate that due to the hydrogen bonding, ethylene adsorption at room temperature also results in strong perturbation of $\mathrm{O}-\mathrm{H}$ stretching vibrations. In this case the low-frequency shift of $\mathrm{OH}$ band to $3200 \mathrm{~cm}^{-1}$ is somewhat larger than for ethylene adsorption by HY. This is well consistent with the higher acidity strength of mordenite in comparison with HY. 
Similar to HY, at the beginning of reaction, much weaker narrow bands from adsorbed ethylene are also clearly seen on the background of the broad band from the hydrogen-bonded hydroxyl groups. However, in accordance with the higher acidity strength of HMOR, ethylene oligomerisation catalyzed by this zeolite is much faster than for HY. Therefore, the DRIFT spectrum of $\pi$-bonded ethylene could be observed only at the very first stage of oligomerization, when the $\pi$-complexes are not yet transformed into ethoxy groups.

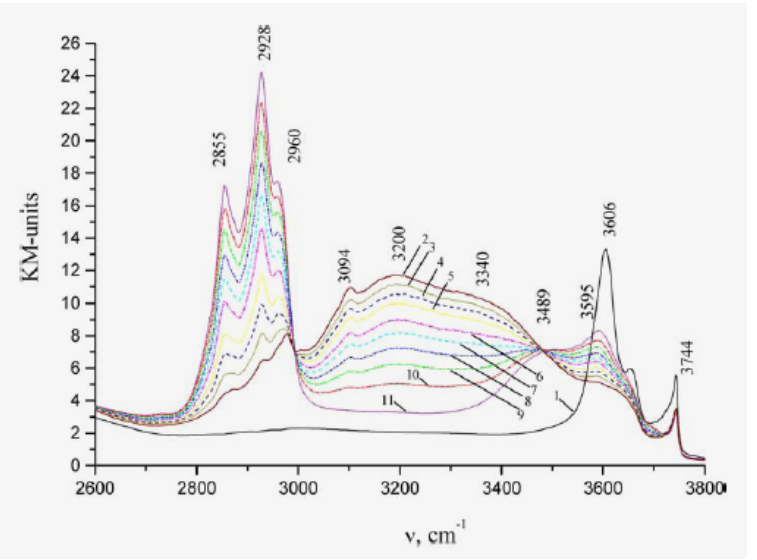

Figure 4: DRIFT spectra of initial HMOR (1) and those recorded after adsorption of ethylene at room temperature in amount of $7 \mathrm{mmol} / \mathrm{g}$ after 0.5 (2); 2.5 (3); 3.5 (4); 4.5 (5); 5.5 (6); 9 (7); 12 (8); 17 (9); 25 (10); and 48 (11) $\mathrm{min}$ after beginning of the reaction. The background created by the zeolite was not subtracted.

At room temperature the broad band from the hydrogen-bonded $\mathrm{OH}$ groups disappears in about 50 minutes after beginning of the reaction. Instead, the intensities of the bands from the growing chains of the oligomer strongly increase. The intensities of the bands at 2855 and at 2928 $\mathrm{cm}^{-1}$ increase with time more rapidly, than the intensity of the band from the asymmetric vibrations of $\mathrm{CH}_{3}$ groups at $2960 \mathrm{~cm}^{-1}$. This indicates a linear structure of the growing oligomer chains.

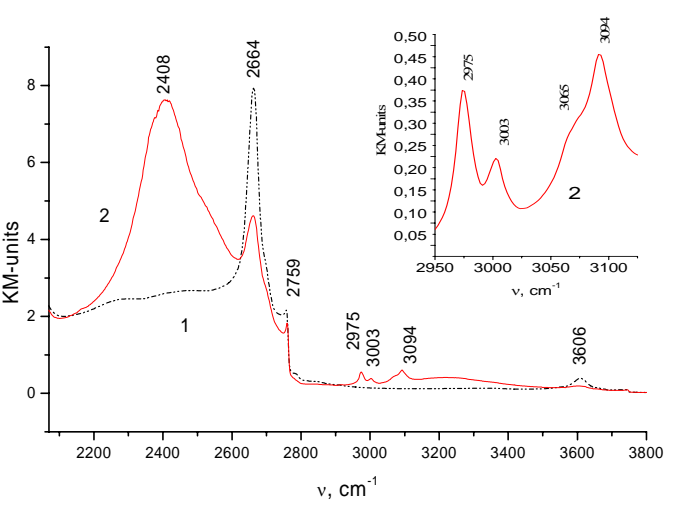

Figure 5: DRIFT spectra of DMOR, recorded before (1) and after (2) adsorption of ethylene in amount of $5 \mathrm{mmol} / \mathrm{g}$ at room temperature. The background created by the zeolite was not subtracted.
To avoid the background created by the hydrogen bonded $\mathrm{OH}$ groups, we studied ethylene adsorption on the deuterated sample. The corresponding DRIFT spectrum is shown in Fig. 5. As one could expect, the low-frequency shift of the band from the hydrogen bonded OD groups is considerably smaller than that for $\mathrm{OH}$ groups. Therefore, for the deuterated sample, the $\mathrm{C}-\mathrm{H}$ stretching bands from adsorbed ethylene are not superimposed on the broad band from the hydrogen bonded OD groups and positions of the $\mathrm{C}-\mathrm{H}$ bands can be detected more precisely. They also only slightly differ from those for ethylene adsorption by HY or by the sodium forms of both zeolites.

In contrast, similar to ethylene adsorption on $\mathrm{HY}$, the intensities of the combinations of the $\mathrm{CH}_{2}$ bending and twisting vibrations with the double bond stretching vibrations in IR spectrum of HMOR also very strongly increase (compare in this connection spectra (a) and (b) in Fig. 1). Despite the positions and shape of the combination bands for HY and HMOR are slightly different, these results indicate that chemical activation of $\pi$-bonded ethylene in mordenite also results in unusually high intensity of the combination band from double bond stretching and $\mathrm{CH}_{2}$ bending and twisting vibrations.

\section{Discussion}

Adsorption of light olefins by hydrogen forms of zeolites has been thoroughly studied by Domen and his coworkers [12-18]. The obtained results indicated that, $\pi$ complexes with acidic protons initially formed upon adsorption, are subsequently involved in the acid catalyzed reactions of double bond shift, skeletal isomerization or oligomerization via formation of carbenium ion-like intermediates or transition states. Selectivity of these reactions depends on temperature and dimensions of zeolites micro pores. For instance, due to narrow micro pores of ferrierite, the main reaction at low temperatures is the double bond shift. For the larger pores in mordenite or ZSM-5 zeolite, predominant reaction at higher temperature is oligomerization, while skeletal isomerization and other secondary reactions require even higher temperatures.

For ethylene the only possible reaction is oligomerization, which does not depend on the pore size. According to the IR $[19,20]$, and solid-state ${ }^{13} \mathrm{C}-\mathrm{NMR}$ studies [21] and to temperature-programmed experiments [22], it starts already at room temperature. The resulting oligomer chains anchored to the surface of the zeolites gradually fill the zeolite micro pores. Decomposition of oligomers ocures above $500 \mathrm{~K}$ resulting in desorption of mixture of hydrocarbons of the similar composition regardless the initial olefin. This temperature is close to that one for the conventional catalytic oligomerisation of ethylene on the corresponding hydrogen forms, which includes the following sequence of elementary steps: 
1. Hydrogen bonding of adsorbed ethylene by the bridging acidic hydroxyl groups:

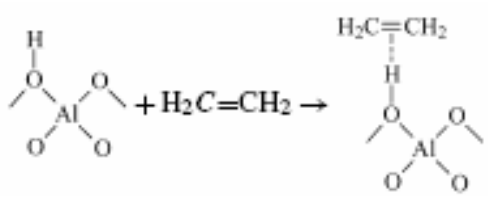

2. Proton transfer to the $\pi$-bonded ethylene resulting in formation of the surface ethoxy groups via carbenium ion like transition state:<smiles>CCOI(OC)(OC)(OC)OC</smiles>

3. Growth of the chains of linear oligomer due to the subsequent reaction of the polarized ethoxy groups (of adsorbed ethyl carbenium ions) with the next ethylene molecules:<smiles></smiles>

4. Desorption of olefins from the zeolite at elevated temperatures via decomposition or cracking of the linear oligomers anchored to the zeolite surface:<smiles>[R7]C=C[R]([H])(OC)[Al](OC)(OC)OCCO[Al](OC)(OC)OC</smiles>

In the present study steps 2 and 3 were directly monitored by DRIFT spectroscopy. However, below we will focus on the reaction (2) that is associated with the activation of hydrogen bonded ethylene via formation of a carbenium ion-like transition state which is subsequently converted into the surface ethoxy group.

Earlier proton transfer from acidic hydroxyl groups of the zeolites to the $\pi$-bonded ethylene has been a subject of our quantum chemical calculations in Refs. [6-7], which indicated that the reaction coordinate of this concerted elementary step is rather complicated. As follows from Fig. 6 that shows the results of these calculations, proton addition to ethylene corresponds to the simultaneous stretching of $\mathrm{O}-\mathrm{H}$ bond of the acidic hydroxyl group in combination with the carbon-carbon double bond stretching. Simultaneously the $\mathrm{H}-\mathrm{C}-\mathrm{H}$ angles in both $\mathrm{CH}_{2}$ fragments of the $\pi$-bonded ethylene are changing due to transformation of one of them into the methyl group and to binding of the other one with the adjacent basic oxygen atom of the zeolite framework. Geometry and the high positive charge of ethyl fragment in such transition state resemble those of adsorbed ethyl carbenium ion. The latter is then converted into the polarized ethoxy group that is covalently bonded to the adjacent oxy- gen atom of the zeolite framework. Later on this reaction mechanism was confirmed by the more sophisticated quantum chemical calculations performed by the other authors [23-26].

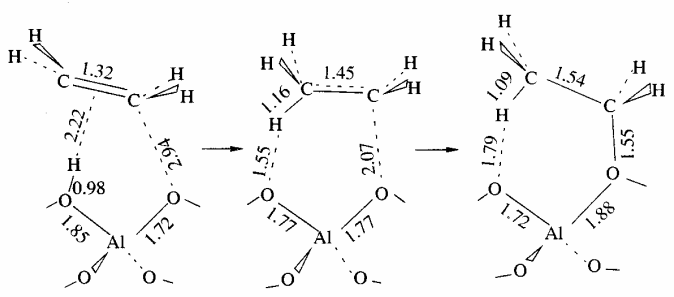

Figure 6: The reaction coordinate of proton addition to of $\pi$-bonded ethylene according to Ref. [ 7 ].

The stretching of the double bond in the $\pi$-bonded complex corresponds to the $v_{3}$ and $v_{2}$ normal vibrations. These vibrations simultaneously change the valence angles in the $\mathrm{CH}_{2}$ groups and, hence, hybridization of the orbitals of the ethylene carbon atoms. The valence angles in both $\mathrm{CH}_{2}$ groups are also influenced by the $v_{10}$ bending vibration. In addition, $v_{4}$ and $v_{7}$ vibrations bring the hydrogen atoms of $\mathrm{CH}_{2}$ groups out of the plain of the ethylene double bond.

According to the above-mentioned quantum chemical calculations, all these vibrations are involved into the reaction coordinate of proton addition to ethylene. The compensation of the energy required for heterolytic dissociation of the bridging hydroxyl group and for the stretching of the double bond upon transformation into the single bond is then supplied by the newly forming $\mathrm{C}-\mathrm{O}$ and $\mathrm{C}-\mathrm{H}$ bonds. Therefore an important feature of proton addition to adsorbed ethylene is a simultaneous rearrangement of several chemical bonds resulting in compensation of the energy required for activation of initial molecules by the energy of the newly forming bonds in the ethoxy fragment. The relatively low activation barrier of this elementary step is favored by the strong polarization of the corresponding combination modes in the $\pi$-bonded molecules.

As follows from the above-discussed DRIFT spectra, the increasing positive charge and dipole moment of $\pi$ bonded ethylene also strongly influences the intensities of IR bands from the corresponding combination vibrations. Unlike intensities of the fundamental IR vibrational bands that are proportional to the squares of the first derivatives of additional dipole moments created by normal vibrations over normal coordinates of these vibrations, intensities of the combination bands are proportional to the squares of the second derivatives of the combination vibrations over their normal coordinates [27]:

$$
\mathrm{I}_{\mathrm{comb}} \sim\left[\mathrm{d}^{2} \mu / \mathrm{dq}^{2}\right]^{2}
$$

Therefore, the very high intensity of the broad IR bands from the combinations of the double bond stretching vibrations with different deformation vibrations of the $\mathrm{CH}_{2}$ groups definitely indicate strong polarization of these com- 
bination vibrations. In contrast, unlike adsorption of light paraffins on the Lewis sites that has been previously studied by us in Refs $[2,3]$, formation of the $\pi$-complexes has practically no influence on intensities of the fundamental bands from $\mathrm{C}-\mathrm{H}$ stretching vibrations. This indicates the absence of polarization of $\mathrm{C}$-H bonds in the $\pi$-bonded species which are not involved into reaction coordinate of proton transfer to adsorbed ethylene.

Thus, according to results of the present study, the previously formulated new spectral criterion for polarization of adsorbed molecules can be also used as a reactivity index for the concerted acid catalyzed oligomerisation of ethylene. For this reaction this criterion should be reformulated in the following way: the strongly polarized vibrations of several chemical bonds involved in the initial stages of the concerted reaction mechanism result in the unusually high intensities of the corresponding combination IR bands.

Most likely this criterion is of more general significance and could be applied as well to other chemical reactions, when the reaction coordinate involves strong polarization of initial molecules resulting from their vibrations. For acid-catalyzed concerted reactions the intensities of the combination IR bands are probably even a better reactivity index than the low frequency shifts of the funda-

\section{References}

[1] L.H.Little, "Infrared spectra of adsorbed molecules", Academic Press 1966, London- New York.

[2] V.B.Kazansky, A.I. Serykh, E.A.Pidko, J.Catal., 225 (2004) 369.

[3] V.B.Kazansky, E.A.Pidko, J. Phys. Chem. B, 109 (2005) 2103.

[4] Liengme B. V., Hall W. K., Trans. Faraday Soc., 62 (1966) 3229 .

[5] A.A.Slinkin, A.V.Kucherov, I.V.Mishin, Kinetics and Catalysis (Russ.), 23 (1982) 1186.

[6] V.B. Kazansky, I. N. Senchenya, J. Catal., 119 (1989) 168.

[7] V.B. Kazansky, I.N. Senchenya, Catal. Lett., 8 (1991) 317.

[8] V.B.Kazansky, Acc. Chem. Res., 24 (1991) 379.

[9] V.B.Kazansky, I.R.Subbotina, R.A. van Santen, E.J.M.Hensen, J. Catal. 227(2004)263.

[10] C.Brecher, R.S.Halford, J. Chem.Phys., 35 (1961) 1109.

[11] Makarova M. A., Zholobenko V.L., Al-Ghefaili Kh. M., et al., J. Chem. Soc. Faraday Trans., 90/7 (1994) 1047.

[12] J.N.Kondo, K Domen, F. Wakabayashi, Microporous and Mesoporous Materials, 21 (1998) 429

[13] J.N.Kondo, E. Yoda, F. Wakabayashi , K Domen, Catal. Today , 63 (2000) 305

[14] J.N.Kondo, K Domen, J. Mol. Catalysis, 199 (2003) 27

[15] E. Yoda, J.N.Kondo, F. Wakabayashi, K Domen, Applied Catalysis A: General, 194-195 (2000) 275

[16] M. Bjorgen, K.-P. Litterud, U. Olsbye, S. Bordiga, A. Zecchina, J. Phys. Chem. B, 108 (2004) 7862 mental stretching bands that are commonly used for this purpose. Indeed, according to results of the present study, intensities of IR bands from the chemical bonds rearranging upon transformation of initially adsorbed molecules into final products increase more than by one order of magnitude, while the corresponding fundamental stretching and combination frequencies decrease only by few percents.

\section{Acknowledgements}

The authors express their thanks for the financial support from the Russian Foundation of the Fundamental Studies (Grant № 04-03-04001), DFG project № 436 RUS 113/778/0-1, NWO Grant № 03-03-89015, the program of the President of the Russian Federation (project 1745.2003.3) and to the program №1 of Department of Chemistry and Material Sciences of Russian Academy of Sciences. They also thank Prof. R. Schlögl for the fruitful discussion of obtained results.
[17] F. Geobaldo, G. Spoto, S. Bordiga, C. Lamberti, A. Zecchina, J. Chem. Soc., Faraday Trans., 93 (1997) 1243

[18] C. Paze, B. Sazak, A. Zecchina, J. Dwyer, J. Phys. Chem. B, 103 (1999) 9978

[19] Cant N.W., Hall W.K., J. Catal., 25 (1972) 161.

[20] G. Spoto, S.Borgia, G. Richiardi, D.Scarano, A. Zecchina, E. Borello, J. Chem Soc. Far. Trans., 90 (1994) 2827.

[21] J. P. van den Berg, J. P. Wolthuizen, A. D. H. Clague, G. R. Hays,R. Huis and J. H. C. van Hooff, J. Catal., 80 (1983) 130.

[22] J. P. van den Berg, J. P. Wolthuizen, and J. H. C. van Hooff, J. Catal., 80 (1983) 139.

[23] P. Viruela - Martin, C.M. Zichovich - Wilson, A. Corma, J. Phys. Chem., 97 (1993) 13713.

[24] R. Blaszkowski en R.A. van Santen, Topics in Catalysis, 4 (1997) 145

[25] M.Boronat, C.M.Cicovich-Wilson, P.M.Viruela, A.Corma, J. Phys. Chem. B, 105 (2001) 1169 .

[26] M.Boronat, P.M.Viruela, A.Corma, J. Am. Chem. Soc., 126 (2004) 3300 .

[27] G. Herzberg, "Molecular Spectra and Molecular Structure II. Infrared and Raman Spectra of Polyatomic Molecules". D. Van. Nostrand Company Inc., Princeton, New York, London 1964. 